\title{
ON THE NUMBER OF REAL ZEROS OF REAL ENTIRE FUNCTIONS WITH A NON-DECREASING SEQUENCE OF THE SECOND QUOTIENTS OF TAYLOR COEFFICIENTS
}

\section{Thu HiEn NGUYen* AND ANNA VishnyakOVA}

Abstract. For an entire function $f(z)=\sum_{k=0}^{\infty} a_{k} z^{k}, a_{k}>0$, we define the sequence of the second quotients of Taylor coefficients $Q:=\left(\frac{a_{k}^{2}}{a_{k-1} a_{k+1}}\right)_{k=1}^{\infty}$. We find new necessary conditions for a function with a non-decreasing sequence $Q$ to belong to the Laguerre-Pólya class of type I. We also estimate the possible number of non-real zeros for a function with a non-decreasing sequence $Q$.

Mathematics subject classification (2020): 30C15, 30D15, 30D35, $26 \mathrm{C} 10$.

Keywords and phrases: Laguerre-Pólya class, Laguerre-Pólya class of type I, entire functions of order zero, real-rooted polynomials, multiplier sequences, complex zero decreasing sequences.

\section{REFERENCES}

[1] A. Bohdanov, Determining bounds on the values of parameters for a function $\varphi_{a}(z, m)=$ $\sum_{k=0}^{\infty} \frac{z^{k}}{a^{k^{2}}}(k !)^{m}, m \in(0,1)$, to Belong to the Laguerre-Pólya Class, Comput. Methods Funct. Theory, 18 (2018), 35-51, doi:10.1007/s40315-017-0210-6.

[2] A. Bohdanov AND A. Vishnyakova, On the conditions for entire functions related to the partial theta-function to belong to the Laguerre-Pólya class, J. Math. Anal. Appl., 434, 2 (2016), 1740-1752, doi:10.1016/j.jmaa.2015.09.084.

[3] T. Craven And G. Csordas, Complex zero decreasing sequences, Methods Appl. Anal., 2 (1995), $420-44$.

[4] G. Gasper and M. Rahman, Basic Hypergeometric Series, Encyclopedia of mathematics and its applications, Cambridge University Press, United Kingdom, Cambridge, 2004.

[5] I. I. Hirschman And D. V. Widder, The Convolution Transform, Princeton University Press, Princeton, New Jersey, 1955.

[6] J. I. Hutchinson, On a remarkable class of entire functions, Trans. Amer. Math. Soc., 25 (1923), 325-332.

[7] O. Katkova, T. Lobova And A. Vishnyakova, On power series having sections with only real zeros, Comput. Methods Funct. Theory, 3, 2 (2003), 425-441.

[8] O. Katkova, T. Lobova And A. Vishnyakova, On entire functions having Taylor sections with only real zeros, J. Math. Phys., Anal., Geom., 11, 4 (2004), 449-469.

[9] V. P. Kostov, On a partial theta function and its spectrum, Proceedings of the Royal Society of Edinburgh Section A: Mathematics, 146, 3 (2016), 609-623.

[10] V. P. Kostov, The closest to 0 spectral number of the partial theta function, C. R. Acad. Bulgare Sci., 69 (2016), 1105-1112.

[11] V. P. Kostov, B. Shapiro, Hardy-Petrovitch-Hutchinson's problem and partial theta function, Duke Math. J., 162, 5 (2013), 825-861.

[12] B. Ja. Levin, Distribution of Zeros of Entire Functions, Transl. Math. Mono., 5, Amer. Math. Soc., Providence, RI, 1964; revised ed. 1980. 
[13] T. H. NGUYEN AND A. VishnYAKOVA, On the entire functions from the Laguerre-Pólya class having the decreasing second quotients of Taylor coefficients, Journal of Mathematical Analysis and Applications, 465, 1 (2018), 348-359, https://doi.org/10.1016/j.jmaa.2018.05.018.

[14] T. H. NGUYen AND A. Vishnyakova, On the necessary condition for entire function with the increasing second quotients of Taylor coefficients to belong to the Laguerre-Pólya class, Journal of Mathematical Analysis and Applications, 480, 2 (2019), https://doi.org/10.1016/j.jmaa.2019.123433.

[15] T. H. NGUYEN AND A. VishnYAKOva, On the closest to zero roots and the second quotients of Taylor coefficients of entire functions from the Laguerre-Pólya I class, Results in Mathematics, 75, 115 (2020), https://doi.org/10.1007/s00025-020-01245-w.

[16] T. H. NGuYen And A. Vishnyakova, On the entire functions from the Laguerre-Pólya I class having the increasing second quotients of Taylor coefficients, Journal of Mathematical Analysis and Applications, 498, 1 (2021), https://doi.org/10.1016/j.jmaa.2021.124955.

[17] T. H. NGUYen, On the conditions for a special entire function related to the partial theta-function and the Euler function to belong to the Laguerre-Pólya class, Computational Methods and Function Theory (2021), https://doi.org/10.1007/s40315-021-00361-0.

[18] N. OBReschKov, Verteilung und Berechnung der Nullstellen reeller Polynome, VEB Deutscher Verlag der Wissenschaften, Berlin, 1963.

[19] I. V. Ostrovs KII, On zero distribution of sections and tails of power series, Israel Math. Conference Proceedings, 15 (2001), 297-310.

[20] G. PÓlya, Collected Papers, Vol. II Location of Zeros, (R. P. Boas ed.) MIT Press, Cambridge, MA, 1974.

[21] G. Pólya AND J. SChur, Uber zwei Arten von Faktorenfolgen in der Theorie der algebraischen Gleichungen, J. Reine Angew. Math., 144 (1914), 89-113.

[22] A. D. SoKAL, The leading root of the partial theta function, Advances in Mathematics, 229, 5 (2012), 2063-2621.

[23] S. O. WARnAar, Partial theta functions, https://www.researchgate.net publication327791878_Partial_theta_functions.

[24] S. O. WarnaAr, Partial Theta Functions. I. Beyond the Lost Notebook, Proc. London Math. Soc., 87, 3 (2003), 363-395. 